\title{
Quaternion and Hopf map characterizations for the existence of rational rotation-minimizing frames on quintic space curves
}

\author{
Rida T. Farouki
}

Received: 13 March 2009 / Accepted: 7 July 2009 /

Published online: 7 August 2009

(C) The Author(s) 2009. This article is published with open access at Springerlink.com

\begin{abstract}
Simple criteria for the existence of rational rotation-minimizing frames (RRMFs) on quintic space curves are determined, in terms of both the quaternion and Hopf map representations for Pythagorean-hodograph (PH) curves in $\mathbb{R}^{3}$. In both cases, these criteria amount to satisfaction of three scalar constraints that are quadratic in the curve coefficients, and are thus much simpler than previous criteria. In quaternion form, RRMF quintics can be characterized by just a single quadratic (vector) constraint on the three quaternion coefficients. In the Hopf map form, the characterization is in terms of one real and one complex quadratic constraint on the six complex coefficients. The identification of these constraints is based on introducing a "canonical form" for spatial PH curves and judicious transformations between the quaternion and Hopf map descriptions. The simplicity of these new characterizations for the RRMF quintics should help facilitate the development of algorithms for their construction, analysis, and practical use in applications such as animation, spatial motion planning, and swept surface constructions.
\end{abstract}

Keywords Rotation-minimizing frames Pythagorean-hodograph curves • Angular velocity $\cdot$ Hopf map • Complex polynomials • Quaternions

Mathematics Subject Classifications (2000) $12 \mathrm{Y} 05$ • 14H45 • 14H50 • 14Q05 • $53 \mathrm{~A} 04 \cdot 68 \mathrm{U} 05 \cdot 68 \mathrm{U} 07$

Communicated by Helmut Pottmann.

R. T. Farouki $(\varangle)$

Department of Mechanical and Aeronautical Engineering,

University of California, Davis, CA 95616, USA

e-mail: farouki@ucdavis.edu 


\section{Introduction}

In motion planning, computer animation, geometric design, and robotics, it is often necessary to specify the variation of an orthonormal frame $\left(\mathbf{f}_{1}, \mathbf{f}_{2}, \mathbf{f}_{3}\right)$ along a given space curve $\mathbf{r}(t)$, that describes the orientation of a rigid body along the given path. The so-called adapted frames on space curves, in which the curve tangent $\mathbf{t}=\mathbf{r}^{\prime} /\left|\mathbf{r}^{\prime}\right|$ is chosen as the frame vector $\mathbf{f}_{1}$, are commonly invoked in such contexts. These frames have one residual freedom, controlling the orientation of the frame vectors $\mathbf{f}_{2}, \mathbf{f}_{3}$ in the curve normal plane.

The Frenet frame $(\mathbf{t}, \mathbf{n}, \mathbf{b})$ - a well-known adapted frame [20]-is often unsuitable for practical applications, since it incurs "unnecessary" rotation of the normal-plane basis vectors $\mathbf{n}, \mathbf{b}$ to ensure that the principal normal $\mathbf{n}$ points towards the center of curvature. As observed by Bishop [1], however, there are infinitely many adapted frames on a given space curve. Klok [19] identified the rotation-minimizing frames (RMFs) as the best suited to swept surface constructions, and he characterized them as solutions of first-order differential equations. Guggenheimer [14] subsequently showed that the RMF normal-plane vectors have an angular orientation relative to the Frenet frame defined, modulo a constant, by the integral of the curve torsion with respect to arc length (which does not ordinarily admit a closed-form reduction). See $[14,16,19,23-25]$ for further details on the applications of RMFs.

The variation of any adapted frame $\left(\mathbf{f}_{1}, \mathbf{f}_{2}, \mathbf{f}_{3}\right)$ with $\mathbf{f}_{1}=\mathbf{t}$ along a curve $\mathbf{r}(t)$ is specified in terms of its vector angular velocity $\omega(t)$ as $^{1}$

$$
\mathbf{f}_{1}^{\prime}=\omega \times \mathbf{f}_{1}, \quad \mathbf{f}_{2}^{\prime}=\omega \times \mathbf{f}_{2}, \quad \mathbf{f}_{3}^{\prime}=\omega \times \mathbf{f}_{3} .
$$

The instantaneous angular speed and rotation axis of the frame $\left(\mathbf{f}_{1}, \mathbf{f}_{2}, \mathbf{f}_{3}\right)$ are specified by the magnitude $\omega=|\omega|$ and direction $\mathbf{a}=\boldsymbol{\omega} /|\boldsymbol{\omega}|$ of the angular velocity. The distinctive property of a rotation-minimizing adapted frame is that its angular velocity has no component along $\mathbf{f}_{1}=\mathbf{t}-$ i.e., $\boldsymbol{\omega} \cdot \mathbf{t} \equiv 0$. In other words, there is no instantaneous rotation of $\mathbf{f}_{2}$ and $\mathbf{f}_{3}$ about $\mathbf{f}_{1}=\mathbf{t}$.

Since polynomial and rational space curves do not ordinarily admit exact rational RMF representation, many authors [12, 16-18, 24] have proposed approximation schemes for them. It was noted in [4] that the Pythagoreanhodograph $(\mathrm{PH})$ curves [5] admit exact RMF computation by integrating a rational function, but in general this incurs transcendental terms. Recently, greater interest in constructing polynomial curves that possess exact rational rotation-minimizing frames (or RRMF curves) has emerged. Such curves are necessarily $\mathrm{PH}$ curves, since only the $\mathrm{PH}$ curves have rational unit tangents, so the problem is to identify constraints on the coefficients of $\mathrm{PH}$ curves that are sufficient and necessary for a rational RMF. Rational forms are always preferable, on account of the exact and efficient computations they permit.

\footnotetext{
${ }^{1}$ In these relations, the curve parameter $t$ is identified with time.
} 
Choi and Han [2] defined a rational adapted frame on spatial PH curves, the so-called Euler-Rodrigues frame (ERF). The ERF is not a geometrically intrinsic frame, since it depends on the chosen coordinate axes. However, it is a more useful reference than the Frenet frame for identifying rational RMFs, on account of its inherently rational nature and its non-singular behavior at inflection points. In [2] the conditions under which the ERF of a PH curve can be an RMF were investigated, and it was shown that: (a) for PH cubics, the ERF and the Frenet frame are equivalent; (b) for PH quintics, the ERF can be an RMF only in the degenerate case of planar curves; and (c) PH curves for which the ERF can be an RMF are of minimum degree 7. Subsequently, Han [15] identified an algebraic criterion characterizing RRMF curves of any (odd) degree, and showed that RRMF cubics are degenerate, i.e., they are either planar PH curves, or PH curves with non-primitive hodographs.

The existence of non-degenerate RRMF quintics was first constructively demonstrated in [9], using the Hopf map representation of spatial PH curves, rather than the more commonly-used quaternion form. The RRMF quintics were characterized by one real and one complex constraint on the six complex coefficients that define the Hopf map form of spatial PH quintics. However, these constraints—of degree 6 and 4 in the coefficients-lack an expected symmetry, and do not easily translate to the quaternion representation.

The goal of the present paper is to remedy these apparent shortcomings of the original characterization of RRMF quintics, presented in [9]. By adopting a special "canonical form" for spatial PH curves, and a sequence of strategic transformations between the Hopf map and quaternion forms, we determine sufficient-and-necessary conditions on the coefficients of spatial PH quintics for the existence of a rational RMF, that are much simpler and more elegant than those determined in [9]. In the Hopf map model, they amount to one real and one complex quadratic constraint; in the quaternion model, a single quadratic (vector) quaternion constraint suffices. In each case, the constraints clearly embody a symmetry that expresses invariance of the RRMF property under the parameter transformation $t \rightarrow 1-t$ applied to $\mathbf{r}(t)$.

The plan for the paper is as follows. After reviewing some basic concepts concerning the quaternion and Hopf map models for spatial PH curves, and their behavior under scaling/rotation transformations in Section 2, a critique of the previous Hopf map form of RRMF conditions [9] is given in Section 3. A "canonical form" for the spatial PH curves is then introduced in Section 4, that greatly simplifies the Hopf map form of the RRMF constraints, and an explicit parameterization of the pairs of complex polynomials satisfying these constraints is identified. Section 5 translates these results into the quaternion model, allowing an elementary relaxation of the canonical-form assumption through the usual interpretation of quaternions as scaling/rotation operators. The general RRMF conditions are translated back into the Hopf map model in Section 6, noting their simplicity and symmetry as compared to the earlier conditions [9]. Finally, Section 7 presents some illustrative examples, while Section 8 summarizes the key results of the paper and identifies interesting avenues for further investigation. 


\section{Quaternion and Hopf map forms}

A polynomial space curve $\mathbf{r}(t)=(x(t), y(t), z(t))$ is a Pythagorean-hodograph $(\mathrm{PH})$ curve if its derivative satisfies

$$
\left|\mathbf{r}^{\prime}(t)\right|=\sqrt{x^{\prime 2}(t)+y^{\prime 2}(t)+z^{\prime 2}(t)}=\sigma(t)
$$

for some polynomial $\sigma(t)$. Two alternative (equivalent) characterizations for spatial hodographs $\mathbf{r}^{\prime}(t)$ satisfying (1) were introduced by Choi et al. [3]. In the quaternion representation, a Pythagorean hodograph is generated from a quaternion polynomial $\mathcal{A}(t)=u(t)+v(t) \mathbf{i}+p(t) \mathbf{j}+q(t) \mathbf{k}$ by the expression

$$
\mathbf{r}^{\prime}(t)=\mathcal{A}(t) \mathbf{i} \mathcal{A}^{*}(t),
$$

$\mathcal{A}^{*}(t)=u(t)-v(t) \mathbf{i}-p(t) \mathbf{j}-q(t) \mathbf{k}$ being the quaternion conjugate of $\mathcal{A}(t)-$ note that the expression on the right is a quaternion with zero real (scalar) part, regarded as a vector in $\mathbb{R}^{3}$. The Hopf map representation, on the other hand, generates spatial Pythagorean hodographs from complex polynomials ${ }^{2}$ $\boldsymbol{\alpha}(t)=u(t)+\mathrm{i} v(t), \boldsymbol{\beta}(t)=q(t)+\mathrm{i} p(t)$ by the expression

$$
\mathbf{r}^{\prime}(t)=\left(|\boldsymbol{\alpha}(t)|^{2}-|\boldsymbol{\beta}(t)|^{2}, 2 \operatorname{Re}(\boldsymbol{\alpha}(t) \overline{\boldsymbol{\beta}}(t)), 2 \operatorname{Im}(\boldsymbol{\alpha}(t) \overline{\boldsymbol{\beta}}(t))\right) .
$$

The equivalence of (2) and (3) may be seen by setting $\mathcal{A}(t)=\boldsymbol{\alpha}(t)+\mathbf{k} \boldsymbol{\beta}(t)$, where the imaginary unit $i$ is identified with the quaternion element $\mathbf{i}$.

A working knowledge of the two representations (2) and (3) - and of the algebra of quaternions, in particular-is necessary to properly appreciate the arguments presented below. For brevity, we shall not re-capitulate here the basic properties, manipulations, and interpretation of quaternion operations. A comprehensive treatment may be found in $[3,5]$ and earlier relevant papers $[2,4,6-13,15,21]$. As a matter of notational convention, we use calligraphic characters to denote quaternions, and often invoke their scalar-vector form [22], denoting these two components of a given quaternion $\mathcal{A}$ by $\operatorname{scal}(\mathcal{A})$ and $\operatorname{vect}(\mathcal{A})$. In expressions where pure scalars or pure vectors are juxtaposed with quaternions, the quaternion product is imputed.

There is an intimate connection between the spatial PH curves and curves with rational rotation-minimizing frames (RRMF curves). Since satisfaction of (1) is necessary for a rational unit tangent, all RRMF curves must be PH curves. Using the quaternion representation, Han [15] has derived a sufficient and necessary condition for a PH curve to possess a rational RMF. Namely, the hodograph (2) defines an RRMF curve if and only if two relatively prime polynomials $a(t), b(t)$ exist, such that the components $u(t), v(t), p(t), q(t)$ of $\mathcal{A}(t)$ satisfy

$$
\frac{u v^{\prime}-u^{\prime} v-p q^{\prime}+p^{\prime} q}{u^{2}+v^{2}+p^{2}+q^{2}}=\frac{a b^{\prime}-a^{\prime} b}{a^{2}+b^{2}} .
$$

\footnotetext{
${ }^{2}$ Bold font symbols are used to denote both complex numbers and vectors in $\mathbb{R}^{3}$ - the meaning should be clear from the context.
} 
Note that the numerator and denominator of the expression on the left can be concisely expressed in terms of $\mathcal{A}(t)$ as $\operatorname{scal}\left(\mathcal{A}(t) \mathbf{i} \mathcal{A}^{* *}(t)\right)$ and $|\mathcal{A}(t)|^{2}$.

In terms of the Hopf map representation, condition (4) can be interpreted [9] as requiring the existence of a complex polynomial $\mathbf{w}(t)=a(t)+\mathrm{i} b(t)$, satisfying $\operatorname{gcd}(a(t), b(t))=$ constant, such that

$$
\frac{\bar{\alpha} \boldsymbol{\alpha}^{\prime}-\overline{\boldsymbol{\alpha}}^{\prime} \boldsymbol{\alpha}+\overline{\boldsymbol{\beta}} \boldsymbol{\beta}^{\prime}-\overline{\boldsymbol{\beta}}^{\prime} \boldsymbol{\beta}}{|\boldsymbol{\alpha}|^{2}+|\boldsymbol{\beta}|^{2}}=\frac{\overline{\mathbf{w}} \mathbf{w}^{\prime}-\overline{\mathbf{w}}^{\prime} \mathbf{w}}{|\mathbf{w}|^{2}} .
$$

In the quaternion representation, a scaling/rotation of the hodograph (2) is effected by the pre- and post- multiplication of $\mathbf{r}^{\prime}(t)$ with a quaternion $\mathcal{S}$ and its conjugate $\mathcal{S}^{*}$ to obtain $\mathcal{S} \mathbf{r}^{\prime}(t) \mathcal{S}^{*}$. This corresponds to the substitution

$$
\mathcal{A}(t) \rightarrow \mathcal{S} \mathcal{A}(t)
$$

in (2). Writing $\mathcal{S}=|\mathcal{S}|\left(\cos \frac{1}{2} \vartheta+\mathbf{a} \sin \frac{1}{2} \vartheta\right)$ where $\mathbf{a}$ is a unit vector, the effect is to scale $\mathbf{r}^{\prime}(t)$ by the factor $|\mathcal{S}|^{2}$ and to rotate it through angle $\vartheta$ about the vector a. Such transformations can likewise be specified in terms of the Hopf map model (3). Writing $\mathcal{A}(t)=\boldsymbol{\alpha}(t)+\mathbf{k} \boldsymbol{\beta}(t)$ and $\mathcal{S}=\rho+\mathbf{k} \boldsymbol{\sigma}$ for suitable complex numbers $\rho, \sigma$ one can verify that the substitution

$$
\left[\begin{array}{l}
\alpha(t) \\
\beta(t)
\end{array}\right] \rightarrow\left[\begin{array}{rr}
\rho(t) & -\bar{\sigma}(t) \\
\sigma(t) & \bar{\rho}(t)
\end{array}\right]\left[\begin{array}{l}
\alpha(t) \\
\beta(t)
\end{array}\right]
$$

in the Hopf map model is equivalent to (6) in the quaternion model: it incurs a scaling of the hodograph (3) by the factor $|\mathcal{S}|^{2}=|\rho|^{2}+|\sigma|^{2}$ and a rotation by angle $\vartheta$ about an axis vector a such that

$$
\cos \vartheta=\frac{\operatorname{Re}(\boldsymbol{\rho})}{\sqrt{|\rho|^{2}+|\boldsymbol{\sigma}|^{2}}}, \quad \sin \vartheta \mathbf{a}=\frac{\operatorname{Im}(\boldsymbol{\rho}) \mathbf{i}+\operatorname{Im}(\boldsymbol{\sigma}) \mathbf{j}+\operatorname{Re}(\boldsymbol{\sigma}) \mathbf{k}}{\sqrt{|\rho|^{2}+|\boldsymbol{\sigma}|^{2}}}
$$

Note that a scaling/rotation transformation of the hodograph does not affect satisfaction of (4) or (5). In the case of (4), the numerator and denominator of $\operatorname{scal}\left(\mathcal{A}(t) \mathbf{i} \mathcal{A}^{\prime *}(t)\right) /|\mathcal{A}(t)|^{2}$ are both multiplied by $|\mathcal{S}|^{2}$ under the map (6). In the case of (5), the numerator and denominator of the left-hand side are both multiplied by $|\rho|^{2}+|\sigma|^{2}$ under the map (7).

Remark 1 In this paper we consider satisfaction of (5) by quintic PH curves defined by quadratic complex polynomials $\boldsymbol{\alpha}(t), \boldsymbol{\beta}(t)$ only when $\mathbf{w}(t)$ is also quadratic (or, equivalently, satisfaction of (4) with $\operatorname{deg}(u(t), v(t), p(t), q(t))=2$ when $\operatorname{deg}(a(t), b(t))=2)$. This fact should be kept in mind in the context of Propositions 1-4 below. The existence of PH quintics satisfying (4) or (5) when $\mathbf{w}(t)=a(t)+\mathrm{i} b(t)$ is of higher degree than 2 remains undecided [9]. 


\section{Hopf map RRMF conditions}

Consider spatial PH quintics specified in the Hopf map representation (3) by quadratic complex polynomials $\boldsymbol{\alpha}(t), \boldsymbol{\beta}(t)$ given in Bernstein form as

$$
\begin{aligned}
& \boldsymbol{\alpha}(t)=\boldsymbol{\alpha}_{0}(1-t)^{2}+\boldsymbol{\alpha}_{1} 2(1-t) t+\boldsymbol{\alpha}_{2} t^{2}, \\
& \boldsymbol{\beta}(t)=\boldsymbol{\beta}_{0}(1-t)^{2}+\boldsymbol{\beta}_{1} 2(1-t) t+\boldsymbol{\beta}_{2} t^{2} .
\end{aligned}
$$

Assuming (see Remark 1) that the polynomial $\mathbf{w}(t)$ in (5) is also quadratic, and written as

$$
\mathbf{w}(t)=\mathbf{w}_{0}(1-t)^{2}+\mathbf{w}_{1} 2(1-t) t+\mathbf{w}_{2} t^{2}
$$

it was shown in [9] that, for some real number $\gamma$, we must have

$$
\begin{aligned}
\left|\boldsymbol{\alpha}_{0}\right|^{2}+\left|\boldsymbol{\beta}_{0}\right|^{2} & =\gamma\left|\mathbf{w}_{0}\right|^{2}, \\
\overline{\boldsymbol{\alpha}}_{0} \boldsymbol{\alpha}_{1}+\overline{\boldsymbol{\beta}}_{0} \boldsymbol{\beta}_{1} & =\gamma \overline{\mathbf{w}}_{0} \mathbf{w}_{1}, \\
\overline{\boldsymbol{\alpha}}_{0} \boldsymbol{\alpha}_{2}+\overline{\boldsymbol{\beta}}_{0} \boldsymbol{\beta}_{2}+2\left(\left|\boldsymbol{\alpha}_{1}\right|^{2}+\left|\boldsymbol{\beta}_{1}\right|^{2}\right) & =\gamma\left(\overline{\mathbf{w}}_{0} \mathbf{w}_{2}+2\left|\mathbf{w}_{1}\right|^{2}\right), \\
\overline{\boldsymbol{\alpha}}_{1} \boldsymbol{\alpha}_{2}+\overline{\boldsymbol{\beta}}_{1} \boldsymbol{\beta}_{2} & =\gamma \overline{\mathbf{w}}_{1} \mathbf{w}_{2}, \\
\left|\boldsymbol{\alpha}_{2}\right|^{2}+\left|\boldsymbol{\beta}_{2}\right|^{2} & =\gamma\left|\mathbf{w}_{2}\right|^{2},
\end{aligned}
$$

in order to satisfy (5). Proposition 2 in [9] established that these equations are consistent if and only if the coefficients of $\boldsymbol{\alpha}(t), \boldsymbol{\beta}(t)$ satisfy the constraints

$$
\left(\left|\boldsymbol{\alpha}_{0}\right|^{2}+\left|\boldsymbol{\beta}_{0}\right|^{2}\right)\left|\overline{\boldsymbol{\alpha}}_{1} \boldsymbol{\alpha}_{2}+\overline{\boldsymbol{\beta}}_{1} \boldsymbol{\beta}_{2}\right|^{2}=\left(\left|\boldsymbol{\alpha}_{2}\right|^{2}+\left|\boldsymbol{\beta}_{2}\right|^{2}\right)\left|\boldsymbol{\alpha}_{0} \overline{\boldsymbol{\alpha}}_{1}+\boldsymbol{\beta}_{0} \overline{\boldsymbol{\beta}}_{1}\right|^{2},
$$

and

$$
\left(\left|\boldsymbol{\alpha}_{0}\right|^{2}+\left|\boldsymbol{\beta}_{0}\right|^{2}\right)\left(\boldsymbol{\alpha}_{0} \boldsymbol{\beta}_{2}-\boldsymbol{\alpha}_{2} \boldsymbol{\beta}_{0}\right)=2\left(\boldsymbol{\alpha}_{0} \overline{\boldsymbol{\alpha}}_{1}+\boldsymbol{\beta}_{0} \overline{\boldsymbol{\beta}}_{1}\right)\left(\boldsymbol{\alpha}_{0} \boldsymbol{\beta}_{1}-\boldsymbol{\alpha}_{1} \boldsymbol{\beta}_{0}\right),
$$

assuming $\boldsymbol{\alpha}_{0} \boldsymbol{\beta}_{1}-\boldsymbol{\alpha}_{1} \boldsymbol{\beta}_{0} \neq 0$, i.e., the curve is not merely a straight line-see [9]. Since condition (10) is real and (11) is complex, these equations amount to three scalar constraints on the coefficients of $\boldsymbol{\alpha}(t), \boldsymbol{\beta}(t)$.

To facilitate derivation of the RRMF conditions (10)-(11), it was observed in Lemma 1 of [9] that, without loss of generality, one may assume $\mathbf{w}_{0}=1$ in (9). However, this assumption incurs an asymmetry in the RRMF conditions. Swapping the 0 and 2 subscripts on the coefficients of $\boldsymbol{\alpha}(t), \boldsymbol{\beta}(t)$ should not alter the RRMF nature of a curve, since this corresponds merely to the reparameterization $t \rightarrow 1-t$. While condition (10) is clearly invariant under such a swap, condition (11) is not. Taking $\mathbf{w}_{2}=1$ rather than $\mathbf{w}_{0}=1$ in (9), we again deduce condition (10), but in lieu of (11) we obtain

$$
\left(\left|\boldsymbol{\alpha}_{2}\right|^{2}+\left|\boldsymbol{\beta}_{2}\right|^{2}\right)\left(\boldsymbol{\alpha}_{0} \boldsymbol{\beta}_{2}-\boldsymbol{\alpha}_{2} \boldsymbol{\beta}_{0}\right)=2\left(\boldsymbol{\alpha}_{2} \overline{\boldsymbol{\alpha}}_{1}+\boldsymbol{\beta}_{2} \overline{\boldsymbol{\beta}}_{1}\right)\left(\boldsymbol{\alpha}_{1} \boldsymbol{\beta}_{2}-\boldsymbol{\alpha}_{2} \boldsymbol{\beta}_{1}\right) .
$$


Note that (12) cannot be deduced from (10) and (11); nor can (11) be deduced from (10) and (12). Equations (11) and (12) together imply that

$$
\frac{\left(\boldsymbol{\alpha}_{0} \overline{\boldsymbol{\alpha}}_{1}+\boldsymbol{\beta}_{0} \overline{\boldsymbol{\beta}}_{1}\right)\left(\boldsymbol{\alpha}_{0} \boldsymbol{\beta}_{1}-\boldsymbol{\alpha}_{1} \boldsymbol{\beta}_{0}\right)}{\left|\boldsymbol{\alpha}_{0}\right|^{2}+\left|\boldsymbol{\beta}_{0}\right|^{2}}=\frac{\left(\boldsymbol{\alpha}_{2} \overline{\boldsymbol{\alpha}}_{1}+\boldsymbol{\beta}_{2} \overline{\boldsymbol{\beta}}_{1}\right)\left(\boldsymbol{\alpha}_{1} \boldsymbol{\beta}_{2}-\boldsymbol{\alpha}_{2} \boldsymbol{\beta}_{1}\right)}{\left|\boldsymbol{\alpha}_{2}\right|^{2}+\left|\boldsymbol{\beta}_{2}\right|^{2}},
$$

and comparing this with (10) then gives

$$
\left(\overline{\boldsymbol{\alpha}}_{0} \boldsymbol{\alpha}_{1}+\overline{\boldsymbol{\beta}}_{0} \boldsymbol{\beta}_{1}\right)\left(\boldsymbol{\alpha}_{1} \boldsymbol{\beta}_{2}-\boldsymbol{\alpha}_{2} \boldsymbol{\beta}_{1}\right)=\left(\overline{\boldsymbol{\alpha}}_{2} \boldsymbol{\alpha}_{1}+\overline{\boldsymbol{\beta}}_{2} \boldsymbol{\beta}_{1}\right)\left(\boldsymbol{\alpha}_{0} \boldsymbol{\beta}_{1}-\boldsymbol{\alpha}_{1} \boldsymbol{\beta}_{0}\right) .
$$

In order to deduce (12), we need (14) in addition to (10) and (11). Conversely, we require (14) to deduce (11), given (10) and (12). This rather cumbersome and asymmetric structure seems unsatisfactory. In Section 6 we shall derive a much simpler Hopf map characterization for the RRMF quintics that is not only inherently symmetric, but also of much lower degree in the coefficients of $\boldsymbol{\alpha}(t), \boldsymbol{\beta}(t)$ than conditions (10)-(12) and (14). Ironically, although we start and end with Hopf map characterizations, the key to identifying this simpler structure involves a detour into the quaternion representation.

\section{Canonical form of spatial PH curves}

The Hopf map characterization of quintic RRMF curves derived in [9] is not very intuitive, and its asymmetric nature seems unfortunate. It was stated in Remark 8 of [9] that the quaternion form, as compared to the Hopf map form, does not seem amenable to deriving "simple" sufficient-and-necessary RRMF conditions. We shall see in Section 5 that this remark was premature, although the Hopf map representation proves instrumental in identifying the quaternion RRMF condition presented below. The other key ingredient is to begin by adopting a certain "canonical form" for the curves under study.

Definition 1 A spatial PH curve $\mathbf{r}(t)=(x(t), y(t), z(t))$ is in canonical form when a coordinate system is chosen such that $\mathbf{r}^{\prime}(0)=(1,0,0)$.

Clearly, any curve in $\mathbb{R}^{3}$ can be mapped to canonical form by means of an appropriate scaling/rotation transformation, as described in Section 2. Note that the polynomials $a(t), b(t)$ satisfying (4)—or, equivalently, the real and imaginary parts of $\mathbf{w}(t)$ in (5) - are invariant under these scaling/rotation transformations. This fact is important, since $a(t), b(t)$ are used to determine the rational RMF from the ERF by a rational rotation-see $[9,15]$.

Remark 2 Since $\mathbf{r}^{\prime}(0)=\left(\left|\boldsymbol{\alpha}_{0}\right|^{2}-\left|\boldsymbol{\beta}_{0}\right|^{2}, 2 \operatorname{Re}\left(\boldsymbol{\alpha}_{0} \overline{\boldsymbol{\beta}}_{0}\right), 2 \operatorname{Im}\left(\boldsymbol{\alpha}_{0} \overline{\boldsymbol{\beta}}_{0}\right)\right)$ we see that $\left|\boldsymbol{\alpha}_{0}\right|=1$ and $\boldsymbol{\beta}_{0}=0$ for a PH curve in canonical form. Moreover, since (3) is unchanged on multiplying $\boldsymbol{\alpha}(t), \boldsymbol{\beta}(t)$ by any unit-modulus complex number $\exp (\mathrm{i} \phi)$, we can take $\boldsymbol{\alpha}_{0}=1$ without loss of generality. 
The assumption of canonical form leads to a significant simplification of the characterization of RRMF quintics in the Hopf map representation (3). It becomes possible to identify explicit forms for the quadratic polynomials $\boldsymbol{\alpha}(t), \boldsymbol{\beta}(t), \mathbf{w}(t)$ satisfying (5), in terms of certain free parameters, that are more convenient than the system of "implicit" constraints on their coefficients derived in [9]. These forms will subsequently be used in Section 5 to derive a remarkably simple quaternion characterization of RRMF quintics.

Proposition 1 A canonical-form spatial PH quintic has a rational RMF if and only if the Bernstein coefficients of the quadratic polynomials $\boldsymbol{\alpha}(t), \boldsymbol{\beta}(t)$ in the Hopf map representation (3) can be expressed in terms of two complex parameters $\zeta, \eta$ and one real parameter $\xi$ in the form

$$
\left(\boldsymbol{\alpha}_{0}, \boldsymbol{\alpha}_{1}, \boldsymbol{\alpha}_{2}\right)=\left(1, \zeta,|\zeta|^{2}-|\boldsymbol{\eta}|^{2}+\mathrm{i} \xi\right), \quad\left(\boldsymbol{\beta}_{0}, \boldsymbol{\beta}_{1}, \boldsymbol{\beta}_{2}\right)=(0, \boldsymbol{\eta}, 2 \overline{\boldsymbol{\zeta}} \boldsymbol{\eta}) .
$$

The Bernstein coefficients of the quadratic polynomial $\mathbf{w}(t)$ in (5) are then

$$
\left(\mathbf{w}_{0}, \mathbf{w}_{1}, \mathbf{w}_{2}\right)=\left(1, \zeta,|\zeta|^{2}+|\eta|^{2}+\mathrm{i} \xi\right) .
$$

Proof By Remark 2, we have $\boldsymbol{\alpha}_{0}=1, \boldsymbol{\beta}_{0}=0$ for a PH quintic in canonical form. Moreover, we can take $\mathbf{w}_{0}=1$ by Lemma 1 in [9]. On setting $\boldsymbol{\alpha}_{1}=\zeta$, $\boldsymbol{\beta}_{1}=\boldsymbol{\eta}$ equations (9) reduce to

$$
\begin{aligned}
1 & =\gamma, \\
\zeta & =\gamma \mathbf{w}_{1}, \\
\boldsymbol{\alpha}_{2}+2\left(|\zeta|^{2}+|\boldsymbol{\eta}|^{2}\right) & =\gamma\left(\mathbf{w}_{2}+2\left|\mathbf{w}_{1}\right|^{2}\right), \\
\bar{\zeta} \boldsymbol{\alpha}_{2}+\bar{\eta} \boldsymbol{\beta}_{2} & =\gamma \overline{\mathbf{w}}_{1} \mathbf{w}_{2}, \\
\left|\boldsymbol{\alpha}_{2}\right|^{2}+\left|\boldsymbol{\beta}_{2}\right|^{2} & =\gamma\left|\mathbf{w}_{2}\right|^{2} .
\end{aligned}
$$

Consider the generic case in which $\zeta, \eta$ are both non-zero. ${ }^{3}$ We have

$$
\gamma=1, \quad \mathbf{w}_{0}=1, \quad \mathbf{w}_{1}=\zeta, \quad \mathbf{w}_{2}=\frac{\bar{\zeta} \boldsymbol{\alpha}_{2}+\overline{\boldsymbol{\eta}} \boldsymbol{\beta}_{2}}{\bar{\zeta}},
$$

the expressions for $\mathbf{w}_{1}$ and $\mathbf{w}_{2}$ coming from the second and fourth equations in (17). Now these values must be consistent with the third and fifth equations in (17). Substituting for $\gamma, \mathbf{w}_{1}, \mathbf{w}_{2}$ into the third equation gives

$$
\boldsymbol{\alpha}_{2}+2\left(|\zeta|^{2}+|\boldsymbol{\eta}|^{2}\right)=\frac{\bar{\zeta} \boldsymbol{\alpha}_{2}+\bar{\eta} \boldsymbol{\beta}_{2}}{\bar{\zeta}}+2|\zeta|^{2},
$$

and by simplification this yields

$$
\boldsymbol{\beta}_{2}=2 \bar{\zeta} \eta
$$

\footnotetext{
${ }^{3}$ See Remark 3 below for the treatment of these special cases.
} 
Substituting for $\gamma, \boldsymbol{\beta}_{2}, \mathbf{w}_{2}$ into the fifth equation then gives

$$
\left|\alpha_{2}\right|^{2}+4|\zeta|^{2}|\eta|^{2}=\frac{\left.\left.\left|\bar{\zeta} \alpha_{2}+2 \bar{\zeta}\right| \eta\right|^{2}\right|^{2}}{|\zeta|^{2}}
$$

Multiplying through by $|\zeta|^{2}$ and expanding the right-hand side yields

$$
|\zeta|^{2}\left|\boldsymbol{\alpha}_{2}\right|^{2}+4|\zeta|^{4}|\eta|^{2}=|\zeta|^{2}\left|\boldsymbol{\alpha}_{2}\right|^{2}+4|\zeta|^{2}|\eta|^{4}+4|\zeta|^{2}|\eta|^{2} \operatorname{Re}\left(\boldsymbol{\alpha}_{2}\right)
$$

and hence we deduce that

$$
\alpha_{2}=|\zeta|^{2}-|\eta|^{2}+\mathrm{i} \xi
$$

where $\xi=\operatorname{Im}\left(\boldsymbol{\alpha}_{2}\right)$ is arbitrary. Finally, substituting from (19) and (20) into expression (18) for $\mathbf{w}_{2}$, we obtain

$$
\mathbf{w}_{2}=\frac{\bar{\zeta}\left(|\zeta|^{2}-|\eta|^{2}+\mathrm{i} \xi\right)+2 \bar{\zeta}|\eta|^{2}}{\bar{\zeta}}=|\zeta|^{2}+|\eta|^{2}+\mathrm{i} \xi .
$$

Hence, the Bernstein coefficients of quadratic polynomials $\boldsymbol{\alpha}(t), \boldsymbol{\beta}(t), \mathbf{w}(t)$ that satisfy (5) are of the form given in (15) and (16).

Remark 3 It was shown in [9] that RRMF quintics degenerate to straight lines when $\boldsymbol{\alpha}_{0} \boldsymbol{\beta}_{1}-\boldsymbol{\alpha}_{1} \boldsymbol{\beta}_{0}=0$. In terms of the coefficients (15), this reduces to $\boldsymbol{\eta}=0$, and (17) then yield $\left(\boldsymbol{\beta}_{0}, \boldsymbol{\beta}_{1}, \boldsymbol{\beta}_{2}\right)=(0,0,0)$ so $\boldsymbol{\beta}(t)$ vanishes and (3) defines a straight line. Furthermore, the condition for degeneration of RRMF quintics to planar curves was identified in [9] as $\operatorname{Im}\left(\overline{\boldsymbol{\alpha}}_{0} \boldsymbol{\alpha}_{1}+\overline{\boldsymbol{\beta}}_{0} \boldsymbol{\beta}_{1}\right)=$ $\operatorname{Im}\left(\overline{\boldsymbol{\alpha}}_{1} \boldsymbol{\alpha}_{2}+\overline{\boldsymbol{\beta}}_{1} \boldsymbol{\beta}_{2}\right)=0$. In terms of the coefficients $(15)$, this gives $\operatorname{Im}(\zeta)=$ $\operatorname{Re}(\zeta) \xi-\left(|\zeta|^{2}+|\eta|^{2}\right) \operatorname{Im}(\zeta)=0$, so either $\zeta=0$ or $\operatorname{Im}(\zeta)=\xi=0$.

For quadratic polynomials $\boldsymbol{\alpha}(t), \boldsymbol{\beta}(t), \mathbf{w}(t)$ with the Bernstein coefficients (15) and (16) one can check that $\overline{\boldsymbol{\alpha}}(t) \boldsymbol{\alpha}^{\prime}(t)-\overline{\boldsymbol{\alpha}}^{\prime}(t) \boldsymbol{\alpha}(t)+\overline{\boldsymbol{\beta}}(t) \boldsymbol{\beta}^{\prime}(t)-\overline{\boldsymbol{\beta}}^{\prime}(t) \boldsymbol{\beta}(t) \equiv$ $\overline{\mathbf{w}}(t) \mathbf{w}^{\prime}(t)-\overline{\mathbf{w}}^{\prime}(t) \mathbf{w}(t)$ by computing Bernstein coefficients of the quadratics on the left and right as

$$
\begin{aligned}
\overline{\boldsymbol{\alpha}}_{0} \boldsymbol{\alpha}_{1}-\overline{\boldsymbol{\alpha}}_{1} \boldsymbol{\alpha}_{0}+\overline{\boldsymbol{\beta}}_{0} \boldsymbol{\beta}_{1}-\overline{\boldsymbol{\beta}}_{1} \boldsymbol{\beta}_{0} & =\overline{\mathbf{w}}_{0} \mathbf{w}_{1}-\overline{\mathbf{w}}_{1} \mathbf{w}_{0}=2 \operatorname{Im}(\zeta) \mathrm{i} \\
\overline{\boldsymbol{\alpha}}_{0} \boldsymbol{\alpha}_{2}-\overline{\boldsymbol{\alpha}}_{2} \boldsymbol{\alpha}_{0}+\overline{\boldsymbol{\beta}}_{0} \boldsymbol{\beta}_{2}-\overline{\boldsymbol{\beta}}_{2} \boldsymbol{\beta}_{0} & =\overline{\mathbf{w}}_{0} \mathbf{w}_{2}-\overline{\mathbf{w}}_{2} \mathbf{w}_{0}=2 \xi \mathrm{i}, \\
\overline{\boldsymbol{\alpha}}_{1} \boldsymbol{\alpha}_{2}-\overline{\boldsymbol{\alpha}}_{2} \boldsymbol{\alpha}_{1}+\overline{\boldsymbol{\beta}}_{1} \boldsymbol{\beta}_{2}-\overline{\boldsymbol{\beta}}_{2} \boldsymbol{\beta}_{1} & =\overline{\mathbf{w}}_{1} \mathbf{w}_{2}-\overline{\mathbf{w}}_{2} \mathbf{w}_{1} \\
& =2 \operatorname{Re}(\zeta) \xi \mathrm{i}-2\left(|\zeta|^{2}+|\boldsymbol{\eta}|^{2}\right) \operatorname{Im}(\zeta) \mathrm{i} .
\end{aligned}
$$


Similarly, $|\boldsymbol{\alpha}(t)|^{2}+|\boldsymbol{\beta}(t)|^{2} \equiv|\mathbf{w}(t)|^{2}$ is verified by computing the Bernstein coefficients of the quartics on the left and right as

$$
\begin{aligned}
& \left|\boldsymbol{\alpha}_{0}\right|^{2}+\left|\boldsymbol{\beta}_{0}\right|^{2}=\left|\mathbf{w}_{0}\right|^{2}=1 \\
& \overline{\boldsymbol{\alpha}}_{0} \boldsymbol{\alpha}_{1}+\overline{\boldsymbol{\alpha}}_{1} \boldsymbol{\alpha}_{0}+\overline{\boldsymbol{\beta}}_{0} \boldsymbol{\beta}_{1}+\overline{\boldsymbol{\beta}}_{1} \boldsymbol{\beta}_{0}=\overline{\mathbf{w}}_{0} \mathbf{w}_{1}+\overline{\mathbf{w}}_{1} \mathbf{w}_{0}=2 \operatorname{Re}(\zeta) \\
& \overline{\boldsymbol{\alpha}}_{0} \boldsymbol{\alpha}_{2}+\overline{\boldsymbol{\alpha}}_{2} \boldsymbol{\alpha}_{0}+\overline{\boldsymbol{\beta}}_{0} \boldsymbol{\beta}_{2}+\overline{\boldsymbol{\beta}}_{2} \boldsymbol{\beta}_{0}+4\left(\left|\boldsymbol{\alpha}_{1}\right|^{2}+\left|\boldsymbol{\beta}_{1}\right|^{2}\right) \\
& =\overline{\mathbf{w}}_{0} \mathbf{w}_{2}+\overline{\mathbf{w}}_{2} \mathbf{w}_{0}+4\left|\mathbf{w}_{1}\right|^{2}=6|\zeta|^{2}+2|\boldsymbol{\eta}|^{2} \\
& \overline{\boldsymbol{\alpha}}_{1} \boldsymbol{\alpha}_{2}+\overline{\boldsymbol{\alpha}}_{2} \boldsymbol{\alpha}_{1}+\overline{\boldsymbol{\beta}}_{1} \boldsymbol{\beta}_{2}+\overline{\boldsymbol{\beta}}_{2} \boldsymbol{\beta}_{1}=\overline{\mathbf{w}}_{1} \mathbf{w}_{2}+\overline{\mathbf{w}}_{2} \mathbf{w}_{1} \\
& =2\left(|\zeta|^{2}+|\eta|^{2}\right) \operatorname{Re}(\zeta)+2 \operatorname{Im}(\zeta) \xi \\
& \left|\boldsymbol{\alpha}_{2}\right|^{2}+\left|\boldsymbol{\beta}_{2}\right|^{2}=\left|\mathbf{w}_{2}\right|^{2}=\left(|\zeta|^{2}+|\boldsymbol{\eta}|^{2}\right)^{2}+\xi^{2}
\end{aligned}
$$

\section{Quaternion RRMF condition}

We now translate canonical-form RRMF quintics, specified in the Hopf map representation, to the quaternion representation. Straightforward arguments then lead to a simple, elegant quaternion characterization of general RRMF quintics. Since prior attempts to determine such a condition using only the quaternion form have failed, these arguments highlight the value of retaining both the forms (2) and (3), and judiciously swapping between them.

In the quaternion representation, a spatial PH quintic is defined through (2) by a quadratic polynomial

$$
\mathcal{A}(t)=\mathcal{A}_{0}(1-t)^{2}+\mathcal{A}_{1} 2(1-t) t+\mathcal{A}_{2} t^{2}
$$

equivalent to the pair of complex quadratic polynomials (8) used in the Hopf map representation, through the relation $\mathcal{A}(t)=\boldsymbol{\alpha}(t)+\mathbf{k} \boldsymbol{\beta}(t)$.

Proposition 2 In terms of the quaternion representation, a canonical-form spatial PH quintic specified by coefficients $\mathcal{A}_{0}=1$ and $\mathcal{A}_{r}=\boldsymbol{\alpha}_{r}+\mathbf{k} \boldsymbol{\beta}_{r}$ for $r=1,2$ in (2) and (21) has a rational $R M F$ if and only if $\mathcal{A}_{2}$ can be expressed in terms of $\mathcal{A}_{1}$ and an arbitrary real number $\xi$ as

$$
\mathcal{A}_{2}=\left(\xi-\mathcal{A}_{1} \mathbf{i} \mathcal{A}_{1}^{*}\right) \mathbf{i} .
$$

Proof In canonical form, we have $\boldsymbol{\alpha}_{0}=1$ and $\boldsymbol{\beta}_{0}=0$, so $\mathcal{A}_{0}=1$. Writing $\boldsymbol{\alpha}_{1}=$ $\boldsymbol{\zeta}=u_{1}+\mathrm{i} v_{1}$ and $\boldsymbol{\beta}_{1}=\boldsymbol{\eta}=q_{1}+\mathrm{i} p_{1}$ gives $\mathcal{A}_{1}=u_{1}+v_{1} \mathbf{i}+p_{1} \mathbf{j}+q_{1} \mathbf{k}$. From (15) we then have

$$
\begin{aligned}
\mathcal{A}_{2} & =\boldsymbol{\alpha}_{2}+\mathbf{k} \boldsymbol{\beta}_{2}=|\zeta|^{2}-|\boldsymbol{\eta}|^{2}+\xi \mathbf{i}+\mathbf{k} 2 \bar{\zeta} \boldsymbol{\eta} \\
& =\left(u_{1}^{2}+v_{1}^{2}-p_{1}^{2}-q_{1}^{2}\right)+\xi \mathbf{i}+2\left(u_{1} p_{1}-v_{1} q_{1}\right) \mathbf{j}+2\left(u_{1} q_{1}+v_{1} p_{1}\right) \mathbf{k} .
\end{aligned}
$$


Noting that $\mathcal{A}_{1} \mathbf{i} \mathcal{A}_{1}^{*}=\left(u_{1}^{2}+v_{1}^{2}-p_{1}^{2}-q_{1}^{2}\right) \mathbf{i}+2\left(u_{1} q_{1}+v_{1} p_{1}\right) \mathbf{j}+2\left(v_{1} q_{1}-u_{1} p_{1}\right) \mathbf{k}$, one can verify that (22) is equivalent to the above expression for $\mathcal{A}_{2}$.

Thus, the $1, \mathbf{i}, \mathbf{j}, \mathbf{k}$ components of $\mathcal{A}_{2}$ are related to those of $\mathcal{A}_{1} \mathbf{i} \mathcal{A}_{1}^{*}$ and $\xi$ by

$$
\left(\mathcal{A}_{2}\right)_{1}=\left(\mathcal{A}_{1} \mathbf{i} \mathcal{A}_{1}^{*}\right)_{\mathbf{i}}, \quad\left(\mathcal{A}_{2}\right)_{\mathbf{i}}=\xi, \quad\left(\mathcal{A}_{2}\right)_{\mathbf{j}}=-\left(\mathcal{A}_{1} \mathbf{i} \mathcal{A}_{1}^{*}\right)_{\mathbf{k}}, \quad\left(\mathcal{A}_{2}\right)_{\mathbf{k}}=\left(\mathcal{A}_{1} \mathbf{i} \mathcal{A}_{1}^{*}\right)_{\mathbf{j}}
$$

To verify that a canonical-form $\mathrm{PH}$ quintic in the quaternion representation obeys (4) when its coefficients satisfy (22), we write

$$
\begin{aligned}
& \mathcal{A}_{0}=1, \quad \mathcal{A}_{1}=u_{1}+v_{1} \mathbf{i}+p_{1} \mathbf{j}+q_{1} \mathbf{k}, \\
& \mathcal{A}_{2}=u_{1}^{2}+v_{1}^{2}-p_{1}^{2}-q_{1}^{2}+\xi \mathbf{i}+2\left(u_{1} p_{1}-v_{1} q_{1}\right) \mathbf{j}+2\left(u_{1} q_{1}+v_{1} p_{1}\right) \mathbf{k},
\end{aligned}
$$

for such a curve. Hence, the quaternion polynomial $\mathcal{A}(t)$ has components of the form

$$
\begin{aligned}
& u(t)=(1-t)^{2}+u_{1} 2(1-t) t+\left(u_{1}^{2}+v_{1}^{2}-p_{1}^{2}-q_{1}^{2}\right) t^{2}, \\
& v(t)=v_{1} 2(1-t) t+\xi t^{2}, \\
& p(t)=p_{1} 2(1-t) t+2\left(u_{1} p_{1}-v_{1} q_{1}\right) t^{2}, \\
& q(t)=q_{1} 2(1-t) t+2\left(u_{1} q_{1}+v_{1} p_{1}\right) t^{2} .
\end{aligned}
$$

Also, from (16) with $\zeta=u_{1}+\mathrm{i} v_{1}$ and $\boldsymbol{\eta}=q_{1}+\mathrm{i} p_{1}$ so that $\mathcal{A}_{1}=\zeta+\mathbf{k} \boldsymbol{\eta}$, we deduce that $a(t)=\operatorname{Re}(\mathbf{w}(t))$ and $b(t)=\operatorname{Im}(\mathbf{w}(t))$ are given by

$$
a(t)=(1-t)^{2}+u_{1} 2(1-t) t+\left|\mathcal{A}_{1}\right|^{2} t^{2}, \quad b(t)=v_{1} 2(1-t) t+\xi t^{2},
$$

where $\left|\mathcal{A}_{1}\right|^{2}=u_{1}^{2}+v_{1}^{2}+p_{1}^{2}+q_{1}^{2}$. Hence, we have

$$
\begin{gathered}
u(t) v^{\prime}(t)-u^{\prime}(t) v(t)-p(t) q^{\prime}(t)+p^{\prime}(t) q(t)=a(t) b^{\prime}(t)-a^{\prime}(t) b(t) \\
=2 v_{1}(1-t)^{2}+\xi 2(1-t) t+2\left(u_{1} \xi-\left|\mathcal{A}_{1}\right|^{2} v_{1}\right) t^{2},
\end{gathered}
$$

and

$$
\begin{aligned}
u^{2}(t) & +v^{2}(t)+p^{2}(t)+q^{2}(t)=a^{2}(t)+b^{2}(t) \\
= & (1-t)^{4}+u_{1} 4(1-t)^{3} t+\left[u_{1}^{2}+v_{1}^{2}+\frac{1}{3}\left(p_{1}^{2}+q_{1}^{2}\right)\right] 6(1-t)^{2} t^{2} \\
& +\left(u_{1}\left|\mathcal{A}_{1}\right|^{2}+v_{1} \xi\right) 4(1-t) t^{3}+\left(\left|\mathcal{A}_{1}\right|^{4}+\xi^{2}\right) t^{4} .
\end{aligned}
$$

In the quaternion representation, the reduction to canonical form of any spatial PH quintic specified through (2) by the quadratic polynomial (21) may be accomplished by pre-multiplying this polynomial with $\mathcal{A}_{0}^{*} /\left|\mathcal{A}_{0}\right|^{2}$, to obtain a quadratic polynomial with coefficients $1, \mathcal{A}_{0}^{*} \mathcal{A}_{1} /\left|\mathcal{A}_{0}\right|^{2}, \mathcal{A}_{0}^{*} \mathcal{A}_{2} /\left|\mathcal{A}_{0}\right|^{2}$. This maps the initial curve derivative $\mathbf{r}^{\prime}(0)=\mathcal{A}_{0} \mathbf{i} \mathcal{A}_{0}^{*}$ into the unit vector $\mathbf{i}$ through a spatial scaling/rotation, and the property of having a rational RMF is clearly invariant under such transformations. This fact can be used to find a simple constraint on the quaternion coefficients $\mathcal{A}_{0}, \mathcal{A}_{1}, \mathcal{A}_{2}$ of spatial PH quintics (in general position) identifying those curves with rational RMFs. 
Proposition 3 A general spatial PH quintic specified by (2) and (21) has a rational $R M F$ if and only if the quaternion coefficients $\mathcal{A}_{0}, \mathcal{A}_{1}, \mathcal{A}_{2}$ satisfy

$$
\operatorname{vect}\left(\mathcal{A}_{2} \mathbf{i} \mathcal{A}_{0}^{*}\right)=\mathcal{A}_{1} \mathbf{i} \mathcal{A}_{1}^{*} .
$$

Proof In the canonical-form condition (22) for RRMF quintics, we imagine the coefficients $\mathcal{A}_{1}, \mathcal{A}_{2}$ to be derived through a scaling/rotation, defined by multiplication with $\mathcal{A}_{0}^{*} /\left|\mathcal{A}_{0}\right|^{2}$, that transforms a PH quintic to canonical form. Substituting $\mathcal{A}_{0}^{*} \mathcal{A}_{1} /\left|\mathcal{A}_{0}\right|^{2}$ and $\mathcal{A}_{0}^{*} \mathcal{A}_{2} /\left|\mathcal{A}_{0}\right|^{2}$ for $\mathcal{A}_{1}$ and $\mathcal{A}_{2}$ in (22), we obtain

$$
\frac{\mathcal{A}_{0}^{*} \mathcal{A}_{2}}{\left|\mathcal{A}_{0}\right|^{2}}=\left[\xi-\frac{\left(\mathcal{A}_{0}^{*} \mathcal{A}_{1}\right)}{\left|\mathcal{A}_{0}\right|^{2}} \mathbf{i} \frac{\left(\mathcal{A}_{0}^{*} \mathcal{A}_{1}\right)^{*}}{\left|\mathcal{A}_{0}\right|^{2}}\right] \mathbf{i},
$$

where the coefficients $\mathcal{A}_{0}, \mathcal{A}_{1}, \mathcal{A}_{2}$ now refer to a general (non-canonical) $\mathrm{PH}$ quintic. Clearing denominators, simplifying, and multiplying both sides on the right with $\mathbf{i}$ then gives

$$
\left|\mathcal{A}_{0}\right|^{2} \mathcal{A}_{0}^{*} \mathcal{A}_{2} \mathbf{i}=\mathcal{A}_{0}^{*} \mathcal{A}_{1} \mathbf{i} \mathcal{A}_{1}^{*} \mathcal{A}_{0}-\left|\mathcal{A}_{0}\right|^{4} \xi .
$$

Multiplying this equation on the left and right by $\mathcal{A}_{0}$ and $\mathcal{A}_{0}^{*}$, and cancelling a common factor $\left|\mathcal{A}_{0}\right|^{4}$, we obtain

$$
\left|\mathcal{A}_{0}\right|^{2} \xi=\mathcal{A}_{1} \mathbf{i} \mathcal{A}_{1}^{*}-\mathcal{A}_{2} \mathbf{i} \mathcal{A}_{0}^{*} .
$$

Since the expression on the left is a pure scalar of arbitrary value- $\xi$ is a free parameter-and $\mathcal{A}_{1} \mathbf{i} \mathcal{A}_{1}^{*}$ is always a pure vector, this equation is evidently equivalent to the stated condition (25).

Remark 4 Unlike the Hopf map characterization of RRMF quintics in [9], the quaternion condition (25) is symmetric, i.e., it is invariant under swapping the 0 and 2 subscripts, corresponding to the re-parameterization $t \rightarrow 1-t$. Namely, since $\left(\mathcal{A}_{2} \mathbf{i} \mathcal{A}_{0}^{*}\right)^{*}=-\mathcal{A}_{0} \mathbf{i} \mathcal{A}_{2}^{*}$, we have $\operatorname{vect}\left(\mathcal{A}_{2} \mathbf{i} \mathcal{A}_{0}^{*}\right)=\operatorname{vect}\left(\mathcal{A}_{0} \mathbf{i} \mathcal{A}_{2}^{*}\right)$.

\section{Symmetric Hopf map RRMF conditions}

In Section 3, we remarked that the Hopf map form of the RRMF conditions derived in [9] do not have the desired property of invariance upon exchanging the 0 and 2 subscripts on the coefficients of the polynomials (8). In Section 4 , a "canonical form" was invoked to determine an explicit parameterization for the set of polynomials (8) satisfying these RRMF conditions. This result was then invoked in Section 5 to identify a simple and symmetric characterization of RRMF quintics in the quaternion representation. Returning to our point of departure, we now use this quaternion condition to identify the symmetric Hopf map RRMF conditions that eluded us in Section 3. 
Proposition 4 A spatial PH quintic specified by the Hopf map form (3) has a rational RMF if and only if the coefficients of the polynomials (8) satisfy

$$
\operatorname{Re}\left(\boldsymbol{\alpha}_{0} \overline{\boldsymbol{\alpha}}_{2}-\boldsymbol{\beta}_{0} \overline{\boldsymbol{\beta}}_{2}\right)=\left|\boldsymbol{\alpha}_{1}\right|^{2}-\left|\boldsymbol{\beta}_{1}\right|^{2}, \quad \boldsymbol{\alpha}_{0} \overline{\boldsymbol{\beta}}_{2}+\boldsymbol{\alpha}_{2} \overline{\boldsymbol{\beta}}_{0}=2 \boldsymbol{\alpha}_{1} \overline{\boldsymbol{\beta}}_{1} .
$$

Proof Writing $\mathcal{A}_{r}=u_{r}+v_{r} \mathbf{i}+p_{r} \mathbf{j}+q_{r} \mathbf{k}$ for $r=0,1,2$ the quaternion RRMF condition (25) is equivalent to the three scalar conditions

$$
\begin{aligned}
& u_{0} u_{2}+v_{0} v_{2}-p_{0} p_{2}-q_{0} q_{2}=u_{1}^{2}+v_{1}^{2}-p_{1}^{2}-q_{1}^{2}, \\
& u_{0} q_{2}+u_{2} q_{0}+v_{0} p_{2}+v_{2} p_{0}=2\left(u_{1} q_{1}+v_{1} p_{1}\right), \\
& v_{0} q_{2}+v_{2} q_{0}-u_{0} p_{2}-u_{2} p_{0}=2\left(v_{1} q_{1}-u_{1} p_{1}\right),
\end{aligned}
$$

and by setting $\boldsymbol{\alpha}_{r}=u_{r}+\mathrm{i} v_{r}$ and $\boldsymbol{\beta}_{r}=q_{r}+\mathrm{i} p_{r}$ for $r=0,1,2$ one can easily verify that these conditions are exactly equivalent to (26).

The Hopf map characterization (26) of the quintic RRMF curves is clearly much simpler and more attractive than the conditions (10)-(11) originally derived in [9]. Unlike the latter, conditions (26) have the desirable property (see Section 3) of invariance if the subscripts 0 and 2 are swapped. Moreover, conditions (26) are quadratic in the coefficients of $\boldsymbol{\alpha}(t), \boldsymbol{\beta}(t)$ while conditions (10) and (11) are degree 6 and 4. Finally, conditions (26) may be interpreted as fixing the middle coefficients $\boldsymbol{\alpha}_{1}, \boldsymbol{\beta}_{1}$ of an RRMF quintic, in terms of the outer coefficients $\boldsymbol{\alpha}_{0}, \boldsymbol{\beta}_{0}, \boldsymbol{\alpha}_{2}, \boldsymbol{\beta}_{2}$ and a free (real) parameter, as follows.

Substituting for $\boldsymbol{\alpha}_{1}$ or $\boldsymbol{\beta}_{1}$ from the second of (26) into the first equation, and writing

$$
P=\operatorname{Re}\left(\boldsymbol{\alpha}_{0} \overline{\boldsymbol{\alpha}}_{2}-\boldsymbol{\beta}_{0} \overline{\boldsymbol{\beta}}_{2}\right), \quad Q=\left|\boldsymbol{\alpha}_{0} \overline{\boldsymbol{\beta}}_{2}+\boldsymbol{\alpha}_{2} \overline{\boldsymbol{\beta}}_{0}\right|,
$$

one can deduce that

$$
\left|\boldsymbol{\alpha}_{1}\right|^{2}=\frac{1}{2}\left(\sqrt{P^{2}+Q^{2}}+P\right), \quad\left|\boldsymbol{\beta}_{1}\right|^{2}=\frac{1}{2}\left(\sqrt{P^{2}+Q^{2}}-P\right) .
$$

The second of (26) then gives

$$
\arg \left(\boldsymbol{\alpha}_{1}\right)-\arg \left(\boldsymbol{\beta}_{1}\right)=\arg \left(\boldsymbol{\alpha}_{0} \overline{\boldsymbol{\beta}}_{2}+\boldsymbol{\alpha}_{2} \overline{\boldsymbol{\beta}}_{0}\right) .
$$

Thus, we may freely assign the argument of $\boldsymbol{\alpha}_{1}$ or $\boldsymbol{\beta}_{1}$, and they are then both determined by (27)-(28). This property will prove advantageous in the construction of RRMF quintics by Hermite interpolation.

It is fairly easy to show that condition (14) follows from (26). First, one can verify that

$$
2\left[\overline{\boldsymbol{\alpha}}_{1} \boldsymbol{\beta}_{1} \boldsymbol{\alpha}_{1}^{2}-\left(\left|\boldsymbol{\alpha}_{1}\right|^{2}-\left|\boldsymbol{\beta}_{1}\right|^{2}\right) \boldsymbol{\alpha}_{1} \boldsymbol{\beta}_{1}-\boldsymbol{\alpha}_{1} \overline{\boldsymbol{\beta}}_{1} \boldsymbol{\beta}_{1}^{2}\right]=0 .
$$

Substituting from (26) for $\overline{\boldsymbol{\alpha}}_{1} \boldsymbol{\beta}_{1},\left|\boldsymbol{\alpha}_{1}\right|^{2}-\left|\boldsymbol{\beta}_{1}\right|^{2}, \boldsymbol{\alpha}_{1} \overline{\boldsymbol{\beta}}_{1}$ into the above gives

$$
\left(\overline{\boldsymbol{\alpha}}_{0} \boldsymbol{\beta}_{2}+\overline{\boldsymbol{\alpha}}_{2} \boldsymbol{\beta}_{0}\right) \boldsymbol{\alpha}_{1}^{2}-2 \operatorname{Re}\left(\boldsymbol{\alpha}_{0} \overline{\boldsymbol{\alpha}}_{2}-\boldsymbol{\beta}_{0} \overline{\boldsymbol{\beta}}_{2}\right) \boldsymbol{\alpha}_{1} \boldsymbol{\beta}_{1}-\left(\boldsymbol{\alpha}_{0} \overline{\boldsymbol{\beta}}_{2}+\boldsymbol{\alpha}_{2} \overline{\boldsymbol{\beta}}_{0}\right) \boldsymbol{\beta}_{1}^{2}=0,
$$

and this is simply the result of expanding and re-arranging (14). 
The fact that conditions (10)-(12) also follow directly from (26) is not so obvious. Consider condition (10) first-by expanding, we obtain

$$
\frac{\left|\overline{\boldsymbol{\alpha}}_{1} \boldsymbol{\alpha}_{2}+\overline{\boldsymbol{\beta}}_{1} \boldsymbol{\beta}_{2}\right|^{2}}{\left|\boldsymbol{\alpha}_{0} \overline{\boldsymbol{\alpha}}_{1}+\boldsymbol{\beta}_{0} \overline{\boldsymbol{\beta}}_{1}\right|^{2}}=\frac{\left|\boldsymbol{\alpha}_{1}\right|^{2}\left|\boldsymbol{\alpha}_{2}\right|^{2}+\left|\boldsymbol{\beta}_{1}\right|^{2}\left|\boldsymbol{\beta}_{2}\right|^{2}+2 \operatorname{Re}\left(\overline{\boldsymbol{\alpha}}_{1} \boldsymbol{\alpha}_{2} \boldsymbol{\beta}_{1} \overline{\boldsymbol{\beta}}_{2}\right)}{\left|\boldsymbol{\alpha}_{0}\right|^{2}\left|\boldsymbol{\alpha}_{1}\right|^{2}+\left|\boldsymbol{\beta}_{0}\right|^{2}\left|\boldsymbol{\beta}_{1}\right|^{2}+2 \operatorname{Re}\left(\boldsymbol{\alpha}_{0} \overline{\boldsymbol{\alpha}}_{1} \overline{\boldsymbol{\beta}}_{0} \boldsymbol{\beta}_{1}\right)} .
$$

Substituting from (27) for $\left|\boldsymbol{\alpha}_{1}\right|^{2},\left|\boldsymbol{\beta}_{1}\right|^{2}$ and $\boldsymbol{\alpha}_{1} \overline{\boldsymbol{\beta}}_{1}=\frac{1}{2}\left(\boldsymbol{\alpha}_{0} \overline{\boldsymbol{\beta}}_{2}+\boldsymbol{\alpha}_{2} \overline{\boldsymbol{\beta}}_{0}\right)$ from the second of equations (26), and simplifying, this ratio becomes

$$
\frac{\left[\sqrt{P^{2}+Q^{2}}+P+2 \operatorname{Re}\left(\boldsymbol{\beta}_{0} \overline{\boldsymbol{\beta}}_{2}\right)\right]\left|\boldsymbol{\alpha}_{2}\right|^{2}+\left[\sqrt{P^{2}+Q^{2}}-P+2 \operatorname{Re}\left(\overline{\boldsymbol{\alpha}}_{0} \boldsymbol{\alpha}_{2}\right)\right]\left|\boldsymbol{\beta}_{2}\right|^{2}}{\left[\sqrt{P^{2}+Q^{2}}+P+2 \operatorname{Re}\left(\overline{\boldsymbol{\beta}}_{0} \boldsymbol{\beta}_{2}\right)\right]\left|\boldsymbol{\alpha}_{0}\right|^{2}+\left[\sqrt{P^{2}+Q^{2}}-P+2 \operatorname{Re}\left(\boldsymbol{\alpha}_{0} \overline{\boldsymbol{\alpha}}_{2}\right)\right]\left|\boldsymbol{\beta}_{0}\right|^{2}}
$$

and by further substituting $P=\operatorname{Re}\left(\boldsymbol{\alpha}_{0} \overline{\boldsymbol{\alpha}}_{2}-\boldsymbol{\beta}_{0} \overline{\boldsymbol{\beta}}_{2}\right)$, this reduces to

$$
\frac{\left[\sqrt{P^{2}+Q^{2}}+\operatorname{Re}\left(\boldsymbol{\alpha}_{0} \overline{\boldsymbol{\alpha}}_{2}+\boldsymbol{\beta}_{0} \overline{\boldsymbol{\beta}}_{2}\right)\right]\left(\left|\boldsymbol{\alpha}_{2}\right|^{2}+\left|\boldsymbol{\beta}_{2}\right|^{2}\right)}{\left[\sqrt{P^{2}+Q^{2}}+\operatorname{Re}\left(\boldsymbol{\alpha}_{0} \overline{\boldsymbol{\alpha}}_{2}+\boldsymbol{\beta}_{0} \overline{\boldsymbol{\beta}}_{2}\right)\right]\left(\left|\boldsymbol{\alpha}_{0}\right|^{2}+\left|\boldsymbol{\beta}_{0}\right|^{2}\right)}=\frac{\left|\boldsymbol{\alpha}_{2}\right|^{2}+\left|\boldsymbol{\beta}_{2}\right|^{2}}{\left|\boldsymbol{\alpha}_{0}\right|^{2}+\left|\boldsymbol{\beta}_{0}\right|^{2}} .
$$

Hence, condition (10) follows from conditions (26).

Consider now condition (11). Expanding the right-hand side gives

$$
2\left(\boldsymbol{\alpha}_{0} \overline{\boldsymbol{\alpha}}_{1}+\boldsymbol{\beta}_{0} \overline{\boldsymbol{\beta}}_{1}\right)\left(\boldsymbol{\alpha}_{0} \boldsymbol{\beta}_{1}-\boldsymbol{\alpha}_{1} \boldsymbol{\beta}_{0}\right)=2\left[\boldsymbol{\alpha}_{0}^{2} \overline{\boldsymbol{\alpha}}_{1} \boldsymbol{\beta}_{1}-\boldsymbol{\beta}_{0}^{2} \boldsymbol{\alpha}_{1} \overline{\boldsymbol{\beta}}_{1}-\left(\left|\boldsymbol{\alpha}_{1}\right|^{2}-\left|\boldsymbol{\beta}_{1}\right|^{2}\right) \boldsymbol{\alpha}_{0} \boldsymbol{\beta}_{0}\right]
$$

and substituting into this from (26) for $\overline{\boldsymbol{\alpha}}_{1} \boldsymbol{\beta}_{1}, \boldsymbol{\alpha}_{1} \overline{\boldsymbol{\beta}}_{1}$, and $\left|\boldsymbol{\alpha}_{1}\right|^{2}-\left|\boldsymbol{\beta}_{1}\right|^{2}$, and writing $\operatorname{Re}\left(\boldsymbol{\alpha}_{0} \overline{\boldsymbol{\alpha}}_{2}-\boldsymbol{\beta}_{0} \overline{\boldsymbol{\beta}}_{2}\right)=\frac{1}{2}\left(\boldsymbol{\alpha}_{0} \overline{\boldsymbol{\alpha}}_{2}-\boldsymbol{\beta}_{0} \overline{\boldsymbol{\beta}}_{2}+\overline{\boldsymbol{\alpha}}_{0} \boldsymbol{\alpha}_{2}-\overline{\boldsymbol{\beta}}_{0} \boldsymbol{\beta}_{2}\right)$, this reduces to

$$
\left|\boldsymbol{\alpha}_{0}\right|^{2} \boldsymbol{\alpha}_{0} \boldsymbol{\beta}_{2}-\left|\boldsymbol{\beta}_{0}\right|^{2} \boldsymbol{\alpha}_{2} \boldsymbol{\beta}_{0}-\left|\boldsymbol{\alpha}_{0}\right|^{2} \boldsymbol{\alpha}_{2} \boldsymbol{\beta}_{0}+\left|\boldsymbol{\beta}_{0}\right|^{2} \boldsymbol{\alpha}_{0} \boldsymbol{\beta}_{2}=\left(\left|\boldsymbol{\alpha}_{0}\right|^{2}+\left|\boldsymbol{\beta}_{0}\right|^{2}\right)\left(\boldsymbol{\alpha}_{0} \boldsymbol{\beta}_{2}-\boldsymbol{\alpha}_{2} \boldsymbol{\beta}_{0}\right) \text {. }
$$

Hence, conditions (26) imply satisfaction of (11). Analogous arguments can be used to show satisfaction of (12).

\section{Computed examples}

Taking the initial and final coefficients of the polynomials (8) as

$$
\boldsymbol{\alpha}_{0}=1+\mathrm{i}, \quad \boldsymbol{\alpha}_{2}=2-\mathrm{i}, \quad \boldsymbol{\beta}_{0}=-1+\mathrm{i}, \quad \boldsymbol{\beta}_{2}=1-2 \mathrm{i},
$$

we have $\boldsymbol{\alpha}_{0} \overline{\boldsymbol{\alpha}}_{2}-\boldsymbol{\beta}_{0} \overline{\boldsymbol{\beta}}_{2}=4+4 \mathrm{i}$ and $\boldsymbol{\alpha}_{0} \overline{\boldsymbol{\beta}}_{2}+\boldsymbol{\alpha}_{2} \overline{\boldsymbol{\beta}}_{0}=-4+2 \mathrm{i}$, and hence

$$
P=\operatorname{Re}\left(\boldsymbol{\alpha}_{0} \overline{\boldsymbol{\alpha}}_{2}-\boldsymbol{\beta}_{0} \overline{\boldsymbol{\beta}}_{2}\right)=4, \quad Q=\left|\boldsymbol{\alpha}_{0} \overline{\boldsymbol{\beta}}_{2}+\boldsymbol{\alpha}_{2} \overline{\boldsymbol{\beta}}_{0}\right|=\sqrt{20} .
$$

Thus, from (27) and (28) we obtain

$$
\left|\boldsymbol{\alpha}_{1}\right|=\sqrt{5}, \quad\left|\boldsymbol{\beta}_{1}\right|=1 \quad \text { and } \quad \arg \left(\boldsymbol{\alpha}_{1}\right)-\arg \left(\boldsymbol{\beta}_{1}\right)=\phi,
$$

where $\phi$ is defined by

$$
\cos \phi=-\frac{2}{\sqrt{5}}, \quad \sin \phi=\frac{1}{\sqrt{5}} .
$$


Thus $\boldsymbol{\alpha}_{1}, \boldsymbol{\beta}_{1}$ may be expressed in terms of a free angular parameter $\theta$ as

$$
\boldsymbol{\alpha}_{1}=\sqrt{5}(\cos \theta+\mathrm{i} \sin \theta), \quad \boldsymbol{\beta}_{1}=\frac{\sin \theta-2 \cos \theta-\mathrm{i}(2 \sin \theta+\cos \theta)}{\sqrt{5}}
$$

One can verify that, for every $\theta$, the complex values (29) and (30) satisfy the Hopf map RRMF conditions (26), with $\operatorname{Re}\left(\boldsymbol{\alpha}_{0} \overline{\boldsymbol{\alpha}}_{2}-\boldsymbol{\beta}_{0} \overline{\boldsymbol{\beta}}_{2}\right)=\left|\boldsymbol{\alpha}_{1}\right|^{2}-\left|\boldsymbol{\beta}_{1}\right|^{2}=4$ and $\boldsymbol{\alpha}_{0} \overline{\boldsymbol{\beta}}_{2}+\boldsymbol{\alpha}_{2} \overline{\boldsymbol{\beta}}_{0}=2 \boldsymbol{\alpha}_{1} \overline{\boldsymbol{\beta}}_{1}=-4+2 \mathrm{i}$.

In terms of the quaternion representation, the choices (29) correspond to

$$
\mathcal{A}_{0}=1+\mathbf{i}+\mathbf{j}-\mathbf{k}, \quad \mathcal{A}_{2}=2-\mathbf{i}-2 \mathbf{j}+\mathbf{k},
$$

and we have $\mathcal{A}_{2} \mathbf{i} \mathcal{A}_{0}^{*}=2+4 \mathbf{i}-4 \mathbf{j}+2 \mathbf{k}$. Hence, the quaternion RRMF condition (25) becomes

$$
\mathcal{A}_{1} \mathbf{i} \mathcal{A}_{1}^{*}=\mathbf{v},
$$

where $\mathbf{v}=4 \mathbf{i}-4 \mathbf{j}+2 \mathbf{k}$. In [8] it was shown that such a quaternion equation has solutions of the form

$$
\mathcal{A}_{1}=\sqrt{|\mathbf{v}|} \mathbf{n} \exp (\phi \mathbf{i})
$$

where

$$
|\mathbf{v}|=6, \quad \mathbf{n}=\frac{\mathbf{v}+|\mathbf{v}| \mathbf{i}}{|\mathbf{v}+| \mathbf{v}|\mathbf{i}|}=\frac{5 \mathbf{i}-2 \mathbf{j}+\mathbf{k}}{\sqrt{30}}, \quad \exp (\phi \mathbf{i})=\cos \phi+\sin \phi \mathbf{i}
$$

with $\phi$ being a free angular parameter. This yields

$$
\mathcal{A}_{1}=-\sqrt{5} \sin \phi+\sqrt{5} \cos \phi \mathbf{i}+\frac{\sin \phi-2 \cos \phi}{\sqrt{5}} \mathbf{j}+\frac{2 \sin \phi+\cos \phi}{\sqrt{5}} \mathbf{k},
$$

and one can easily verify that, with the choice $\phi=\theta-\frac{1}{2} \pi$, this is equal to $\alpha_{1}+$ $\mathbf{k} \boldsymbol{\beta}_{1}$ when $\boldsymbol{\alpha}_{1}$ and $\boldsymbol{\beta}_{1}$ are given by (30).

The quaternion representation can be reduced to canonical form through a pre-multiplication of $\mathcal{A}_{0}, \mathcal{A}_{1}, \mathcal{A}_{2}$ with $\mathcal{A}_{0}^{*} /\left|\mathcal{A}_{0}\right|^{2}$ to obtain the new coefficients

$$
\begin{aligned}
& \mathcal{A}_{0}=1, \quad \mathcal{A}_{2}=-\frac{1}{2}-\frac{1}{2} \mathbf{i}-\mathbf{j}+\mathbf{k} \\
& \mathcal{A}_{1}=\frac{1}{2 \sqrt{5}}[-3 \sin \phi+\cos \phi+(\sin \phi+3 \cos \phi) \mathbf{i} \\
&\quad+(4 \sin \phi+2 \cos \phi) \mathbf{j}+(-2 \sin \phi+4 \cos \phi) \mathbf{k}] .
\end{aligned}
$$




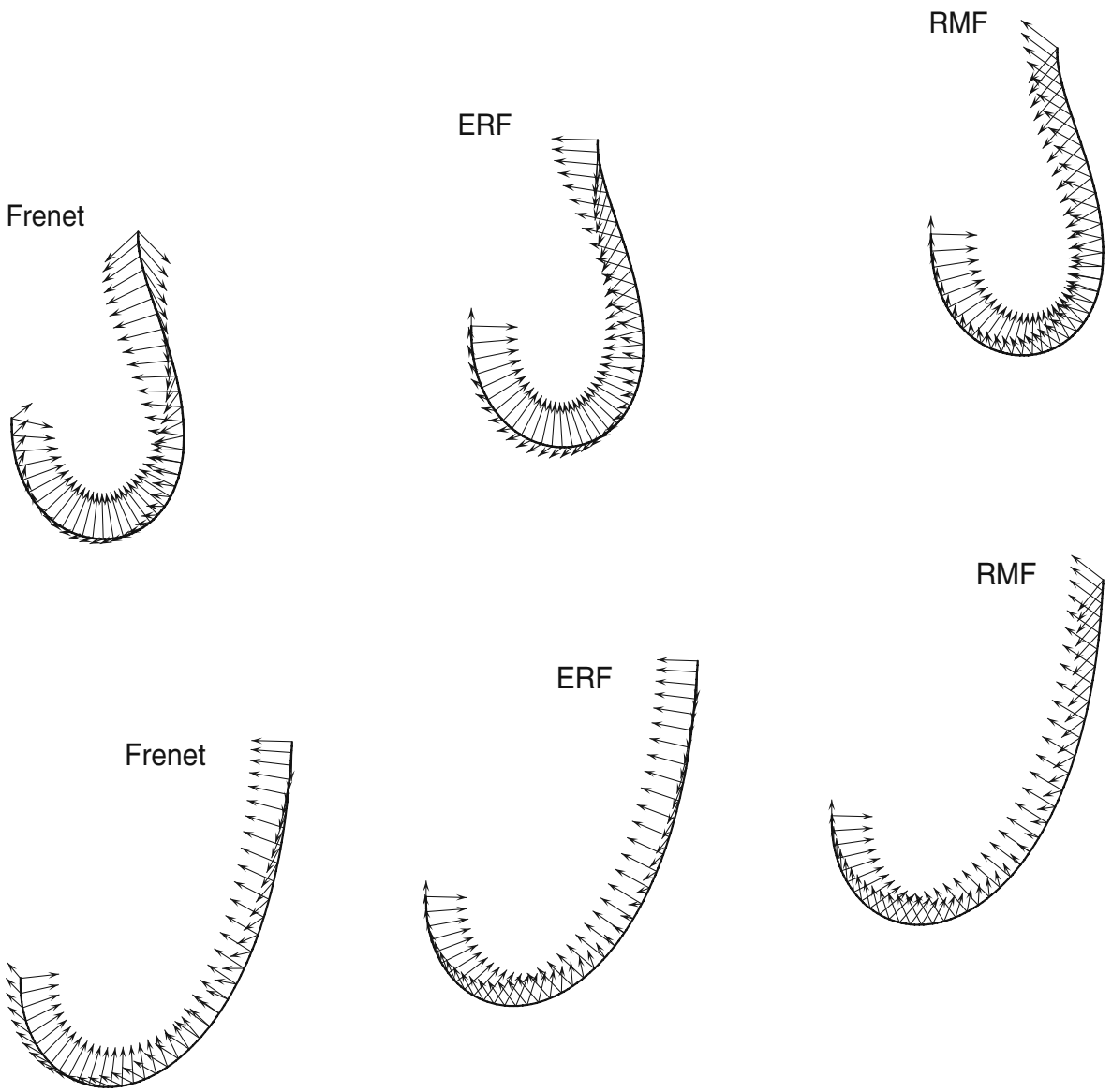

Fig. 1 The RRMF quintics determined by the quaternion coefficients (31) with $\phi=0$ (upper curve) and $\phi=-\frac{1}{4} \pi$ (lower curve), illustrating the Frenet frame (left), the Euler-Rodrigues frame (center), and the rational rotation-minimizing frame (right). For clarity, the unit tangent vector (common to all three adapted frames) is omitted: only the normal-plane vectors are shown

Comparing these coefficients with (21) for the canonical-form components (23) of $\mathcal{A}(t)=u(t)+v(t) \mathbf{i}+p(t) \mathbf{j}+q(t) \mathbf{k}$ gives the correspondence

$$
\begin{aligned}
& u_{1}=\frac{-3 \sin \phi+\cos \phi}{2 \sqrt{5}}, \quad v_{1}=\frac{\sin \phi+3 \cos \phi}{2 \sqrt{5}}, \\
& p_{1}=\frac{2 \sin \phi+\cos \phi}{\sqrt{5}}, \quad q_{1}=\frac{-\sin \phi+2 \cos \phi}{\sqrt{5}}, \quad \xi=-\frac{1}{2} .
\end{aligned}
$$

Hence, noting that $\left|\mathcal{A}_{1}\right|^{2}=\frac{3}{2}$, we can identify the two polynomials (24) that determine the RRMF through a rational rotation of the ERF as

$$
a(t)=(1-t)^{2}+u_{1} 2(1-t) t+\frac{3}{2} t^{2}, \quad b(t)=v_{1} 2(1-t) t-\frac{1}{2} t^{2},
$$


where $u_{1}, v_{1}$ are as defined above-see [9] for details on the computation of the rational RMF from the ERF and $a(t), b(t)$.

The curve control points can be expressed [7] in terms of $\mathcal{A}_{0}, \mathcal{A}_{1}, \mathcal{A}_{2}$ as

$$
\begin{aligned}
& \mathbf{p}_{1}=\mathbf{p}_{0}+\frac{1}{5} \mathcal{A}_{0} \mathbf{i} \mathcal{A}_{0}^{*}, \\
& \mathbf{p}_{2}=\mathbf{p}_{1}+\frac{1}{10}\left(\mathcal{A}_{0} \mathbf{i} \mathcal{A}_{1}^{*}+\mathcal{A}_{1} \mathbf{i} \mathcal{A}_{0}^{*}\right), \\
& \mathbf{p}_{3}=\mathbf{p}_{2}+\frac{1}{30}\left(\mathcal{A}_{0} \mathbf{i} \mathcal{A}_{2}^{*}+4 \mathcal{A}_{1} \mathbf{i} \mathcal{A}_{1}^{*}+\mathcal{A}_{2} \mathbf{i} \mathcal{A}_{0}^{*}\right), \\
& \mathbf{p}_{4}=\mathbf{p}_{3}+\frac{1}{10}\left(\mathcal{A}_{1} \mathbf{i} \mathcal{A}_{2}^{*}+\mathcal{A}_{2} \mathbf{i} \mathcal{A}_{1}^{*}\right), \\
& \mathbf{p}_{5}=\mathbf{p}_{4}+\frac{1}{5} \mathcal{A}_{2} \mathbf{i} \mathcal{A}_{2}^{*},
\end{aligned}
$$

$\mathbf{p}_{0}$ being an arbitrary integration constant. Figure 1 illustrates this RRMF quintic, together with its Frenet frame, ERF, and RMF, for two values of $\phi$.

\section{Closure}

Adopting canonical coordinates and freely switching between the Hopf map and quaternion representations of spatial PH quintics yields greatly simplified constraints identifying the family of curves with rational rotation-minimizing frames (RRMF quintics). These constraints are merely quadratic in the curve coefficients, and exhibit the desirable symmetry property of invariance under a reversion of the curve parameter. The relatively simple nature of these new RRMF constraints - compared to those formerly available-should greatly facilitate development of algorithms for the construction, shape analysis, and practical applications of quintic RRMF curves. It is expected that these new, simplified characterizations will pave the way toward widespread utilization of the RRMF curves in motion planning, computer animation, swept surface constructions, robotics, and other fields in which the specification/control of spatial orientation along a given path is of central concern.

Open Access This article is distributed under the terms of the Creative Commons Attribution Noncommercial License which permits any noncommercial use, distribution, and reproduction in any medium, provided the original author(s) and source are credited.

\section{References}

1. Bishop, R.L.: There is more than one way to frame a curve. Am. Math. Mo. 82, 246-251 (1975)

2. Choi, H.I., Han, C.Y.: Euler-Rodrigues frames on spatial Pythagorean-hodograph curves. Comput. Aided Geom. Des. 19, 603-620 (2002)

3. Choi, H.I., Lee, D.S., Moon, H.P.: Clifford algebra, spin representation, and rational parameterization of curves and surfaces. Adv. Comp. Math. 17, 5-48 (2002)

4. Farouki, R.T.: Exact rotation-minimizing frames for spatial Pythagorean-hodograph curves. Graph. Models 64, 382-395 (2002)

5. Farouki, R.T.: Pythagorean-Hodograph Curves: Algebra and Geometry Inseparable. Springer, Berlin (2008) 
6. Farouki, R.T., al-Kandari, M., Sakkalis, T.: Structural invariance of spatial Pythagorean hodographs. Comput. Aided Geom. Des. 19, 395-407 (2002)

7. Farouki, R.T., al-Kandari, M., Sakkalis, T. Hermite interpolation by rotation-invariant spatial Pythagorean-hodograph curves. Adv. Comp. Math. 17, 369-383 (2002)

8. Farouki, R.T., Giannelli, C., Manni, C., Sestini, A.: Identification of spatial PH quintic Hermite interpolants with near-optimal shape measures. Comput. Aided Geom. Des. 25, 274-297 (2008)

9. Farouki, R.T., Giannelli, C., Manni, C., Sestini, A.: Quintic space curves with rational rotationminimizing frames. Comput. Aided Geom. Des. 26, 580-592 (2009)

10. Farouki, R.T., Giannelli, C., Sestini, A.: Helical polynomial curves and double Pythagorean hodographs I. Quaternion and Hopf map representations. J. Symb. Comput. 44, 161-179 (2009)

11. Farouki, R.T., Giannelli, C., Sestini, A.: Helical polynomial curves and double Pythagorean hodographs II. Enumeration of low-degree curves. J. Symb. Comput. 44, 307-332 (2009)

12. Farouki, R.T., Han, C.Y.: Rational approximation schemes for rotation-minimizing frames on Pythagorean-hodograph curves. Comput. Aided Geom. Des. 20, 435-454 (2003)

13. Farouki, R.T., Han, C.Y., Manni, C., Sestini, A.: Characterization and construction of helical polynomial space curves. J. Comput. Appl. Math. 162, 365-392 (2004)

14. Guggenheimer, H.: Computing frames along a trajectory. Comput. Aided Geom. Des. 6, 77-78 (1989)

15. Han, C.Y.: Nonexistence of rational rotation-minimizing frames on cubic curves. Comput. Aided Geom. Des. 25, 298-304 (2008)

16. Jüttler, B.: Generating rational frames of space curves via Hermite interpolation with Pythagorean hodograph cubic splines. In: Geometric Modelling and Processing '98, pp. 83106. Bookplus, Seoul (1998)

17. Jüttler, B., Mäurer, C.: Cubic Pythagorean hodograph spline curves and applications to sweep surface modelling. Comput. Aided Des. 31, 73-83 (1999)

18. Jüttler, B., Mäurer, C.: Rational approximation of rotation minimizing frames using Pythagorean-hodograph cubics. J. Geom. Graphics 3, 141-159 (1999)

19. Klok, F.: Two moving coordinate frames for sweeping along a 3D trajectory. Comput. Aided Geom. Des. 3, 217-229 (1986)

20. Kreyszig, E.: Differential Geometry. University of Toronto Press (1959)

21. Monterde, J.: A characterization of helical polynomial curves of any degree. Adv. Comp. Math. 30, 61-78 (2009)

22. Roe, J.: Elementary Geometry. Oxford University Press, Oxford (1993)

23. Sír, Z., Jüttler, B.: Spatial Pythagorean hodograph quintics and the approximation of pipe surfaces. In: Martin, R., Bez, H., Sabin, M. (eds.) Mathematics of Surfaces XI, pp. 364-380. Springer, Berlin (2005)

24. Wang, W., Joe, B.: Robust computation of the rotation minimizing frame for sweep surface modelling. Comput. Aided Des. 29, 379-391 (1997)

25. Wang, W., Jüttler, B., Zheng, D., Liu, Y.: Computation of rotation minimizing frames. ACM Trans. Graphics 27(1), 1-18, Article 2 (2008) 